Nina L. Tamashunas, BS

Department of Dermatology, Cleveland Clinic, Cleveland, $\mathrm{OH}$; Case Western Reserve University School of Medicine, Cleveland, $\mathrm{OH}$
Wilma F. Bergfeld, MD, FAAD

Senior Dermatologist, and Director, Dermatopathology

Fellowship, Departments of Dermatology and Pathology,

Cleveland Clinic, Cleveland, OH; Past President, American

Academy of Dermatology, American Society of Dermato-

pathology, and the American Dermatologic Association

\title{
Male and female pattern hair loss: Treatable and worth treating
}

\section{ABSTRACT}

Pattern hair loss is the most common type of hair loss in both men and women. Scalp hair is typically affected in a characteristic distribution without other scalp or dermatologic findings. Early recognition and treatment can help halt its progression to preserve as much hair as possible. Both pharmacologic and nonpharmacologic treatments have proven helpful.

\section{KEY POINTS}

Male and female pattern hair loss is a nonscarring, progressive form of alopecia that typically affects the temporal, frontal, and vertex scalp in men and central scalp in women.

The process can begin soon after puberty, and the resulting hair loss negatively affects quality of life and selfimage.

Pattern hair loss is commonly diagnosed with a thorough history; physical examination of the face, scalp, and nails; the hair-pull test; dermoscopy; and laboratory testing. A hair biopsy may be of value for clinically challenging cases.

Topical minoxidil and oral finasteride are first-line treatments for male pattern hair loss and topical minoxidil is the first-line therapy for female pattern hair loss, but there are a number of other off-label pharmacologic and nonpharmacologic treatments.
ATTERn haIR loss is a progressive, nonscarring form of hair loss characterized by gradual loss of terminal hair and follicular miniaturization to vellus hair fibers on the scalp in a characteristic distribution. It is the most common form of hair loss in both men and women and has psychosocial effects, including stress and diminished quality of life.

This review focuses on clinical presentation, diagnosis, and treatment of pattern hair loss.

\section{MANY NAMES FOR IT}

This condition goes by many names, such as androgenetic alopecia, androgenic alopecia, male balding, male pattern hair loss, female pattern alopecia, diffuse alopecia in women, and hereditary alopecia. The term "androgenetic alopecia" was used in the past, recognizing the hormonal and hereditary influences underlying the condition in men.

As our understanding of both the pathophysiology and phenotypic expression expanded, so did the collection of terms used to identify this disorder. Newer terminology developed to express the different patterns of presentation in men and women and the uncertain role, and frequent absence, of androgen excess in women. Male pattern hair loss and female pattern hair loss are now the favored terms.

\section{GENES PLAY A ROLE}

Male and female pattern hair loss are polygenic conditions, which explains their high prevalence and variable phenotypic expression. ${ }^{1}$ Epigenetic modifications may alter genetic susceptibility. ${ }^{1}$

Interestingly, genetic variations associated with the androgen receptor gene $(A R)$ have 



Figure 1. Male pattern hair loss.

been linked to development of male pattern hair loss, but genes for aromatase (CYP19A1), estrogen receptor-a (ESR1), type I 5-alpha reductase (SRD5A1), and insulin-like growth factor 2 (IGF-2) do not have any established association with it. ${ }^{1}$

Research into genetic associations with female pattern hair loss is less extensive and robust than that of male pattern hair loss. Studying the relationship between female pattern hair loss and $A R$ has proven difficult, since $A R$ is located on the $\mathrm{X}$ chromosome, which undergoes $\mathrm{X}$ inactivation in women. ${ }^{1}$ An allelic variant of CYP19A1 was associated with a predisposition to female pattern hair loss in a genome-wide association study. ${ }^{2}$

\section{LINKED TO ANDROGEN EXCESS IN MEN}

Androgens are considered necessary for male pattern hair loss to develop. The condition typically begins after the start of puberty, which is marked by a striking increase in androgen levels. Dihydroxytestosterone, a potent metabolite of testosterone synthesized in a reaction catalyzed by 5 -alpha reductase in the peripheral target organs, hair follicle, and sebaceous glands, plays a role in normal hair growth and male pattern hair loss development in androgen-sensitive areas such as the vertex and frontal scalp, beard, axilla, pubis, and extremities. Dihydroxytestosterone assists normal hair growth in these areas, but elevated cellular levels of androgen receptors and 5-alpha reductase ${ }^{3}$ and increased production of dihydroxytestosterone ${ }^{4}$ have been documented in cases of male pattern hair loss. No cases of male pattern hair loss have been documented in men with 5-alpha reductase deficiencies. ${ }^{5}$

\section{UNCLEAR RELATIONSHIP WITH HORMONES IN WOMEN}

The relationship between androgens and female pattern hair loss is less clear. Female pattern hair loss has been observed in women with high androgen levels, ${ }^{6}$ but it has also been documented in a patient with complete androgen insensitivity syndrome. ${ }^{7}$ Additionally, most women with female pattern hair loss have normal testosterone levels and lack clinical manifestations of hyperandrogenemia. ${ }^{6}$

The role of circulating estrogens in the development of female pattern hair loss is also unclear. The prevalence of hair loss increases after menopause. Evidence is conflicting regarding whether estrogen stimulates or inhibits the hair follicle. ${ }^{1}$

\section{CAN BEGIN EARLY}

Pattern hair loss in men and women begins soon after puberty. Thinning of hair and nonscarring loss of terminal hairs, resulting in a decrease in hair density, generally progress slowly over years. The scalp is healthy without associated symptoms.

In men, hair loss typically affects the central scalp, including the midfrontal, temporal, and vertex regions (Figure 1). The 7-stage Hamilton-Norwood scale is commonly used 
to classify male pattern hair loss. ${ }^{8}$ However, in some men, hair loss does not follow this typical progression or is more severe in particular areas.

In women, the characteristic distribution of hair loss is different. Female pattern hair loss has 2 general distributions: diffuse thinning across the central scalp and the characteristic "Christmas tree" pattern observed along the midline part of the hair due to prominent hair thinning towards the front of the scalp with minimal involvement of the hairline (Figure 2). ${ }^{9,10}$ The frontal hairline is less likely to be involved, but bitemporal thinning is common. The 3-grade Ludwig scale is commonly used to characterize female pattern hair loss. ${ }^{11}$

\section{A CLINICAL DIAGNOSIS}

Pattern hair loss is typically diagnosed clinically (Table 1 ).

\section{History}

A thorough history should be elicted, including age of onset of hair loss, time course, severity, hair loss distribution, progression (ie, periods of shedding), and accompanying symptoms. For women, a gynecologic history may help uncover an underlying cause such as polycystic ovarian syndrome or hyperandrogenism. The patient should be asked about any family history of hair loss, metabolic syndromes (eg, diabetes mellitus), and androgen excess; medications; and medical history.

Conditions that worsen hair loss, including iron deficiency, thyroid dysfunction, and nutritional deficiencies, should be considered and managed to improve treatment results.

\section{Physical examination}

A complete skin evaluation should be conducted, including the face, scalp, and nails.

When examining the scalp, note the distribution of hair loss, the caliber of hairs, and other clinical features. Male pattern hair loss typically presents as a receding hairline and hair miniaturization on the frontal and vertex scalp. In women, the vertex and midfrontal scalp are commonly affected, as described above. Hair loss can be assessed by comparing the hair part of the central scalp with that of the occipital scalp, which is generally spared. Hair miniaturization can be seen better using
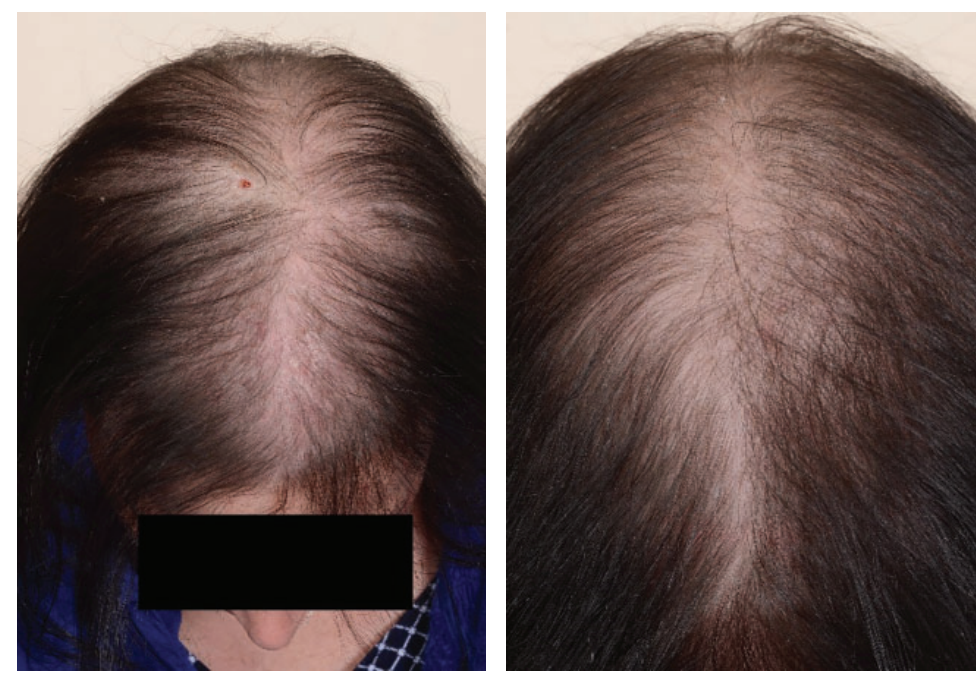

Figure 2. Female pattern hair loss.

a sheet of paper as a backdrop and comparing the caliber of adjacent hair shafts.

Inflammation, scarring, or scaling of the scalp suggests a different diagnosis, as pattern hair loss is usually unaccompanied by these signs. Nevertheless, seborrheic dermatitis is more prevalent in people with pattern hair loss, ${ }^{12}$ so male and female pattern hair loss can present with another scalp condition. Seborrheic dermatitis is often associated with seborrhea (oily scalp) which is a result of androgen stimulation of the sebaceous glands.

Nail involvement (eg, pitting, trachyonychia, and longitudinal ridging) and patchy hair loss in nonscalp regions (eg, the eyebrows) are inconsistent with the diagnosis of male or female pattern hair loss.

\section{Hair-pull test}

The hair-pull test, which is useful in detecting active hair loss, is performed by grasping 50 to 60 hairs close to the scalp with the thumb, index, and middle fingers and slowly pulling. If 6 or more hairs come loose, hair loss is likely active.

Extracted hair can be examined under the microscope to characterize the type (eg, broken or dystrophic) and the phase (eg, telogen [resting] or anagen [growth]). A study by McDonald et $\mathrm{al}^{13}$ suggested that neither washing nor brushing the hair affects results of the hair-pull test. In pattern hair loss, the hairpull test is generally negative, though it can be positive early in the process on the vertex or midfrontal scalp. 


\section{Dermoscopy}

Examination of the scalp with a dermatoscope can reveal epidermal and dermal structures undetectable with the naked eye. Dermoscopic findings of diversity in hair diameter, yellow dots (sebaceous glands), perifollicular pigmentation, and lack of scarring are consistent with the diagnosis of male or female pattern hair loss. Small focal areas with complete hair loss may be observed, and skin pigmentation in these areas may vary due to sun exposure. ${ }^{14}$

\section{Scalp biopsy}

Though generally not required, a scalp biopsy can be helpful when the clinical picture is unclear or coexisting scalp conditions are suspected. Two 4-mm punch biopsies are taken in the direction of the hair shaft, allowing for transverse and vertical sectioning.

Histologic features of male and female pattern hair loss include terminal hair miniaturization (hair shaft diameter $\leq 0.03 \mathrm{~mm}$ ), increased percentage of telogen hairs $(15 \%-$ $20 \%$ ), decreased ratio of terminal to vellus or vellus-like hairs (1.9:1 in men and 1.5:1 in women), and reduced total number of hairs per unit area. ${ }^{10}$

\section{Androgens are considered necessary for male pattern hair loss}

\section{Laboratory testing}

Thyroid-stimulating hormone and iron studies (including serum ferritin, serum iron, and total iron binding capacity) can be helpful in assessing men and women with pattern hair loss. ${ }^{10}$

Women with clinical manifestations of androgen excess such as hirsutism, adult acne, irregular menses, and acanthosis nigricans should undergo a laboratory workup for hyperandrogenemia. ${ }^{10}$ This includes free or total testosterone with or without dehydroepiandrosterone sulfate. ${ }^{10}$ Measuring serum prolactin can also be considered for women presenting with concomitant galactorrhea or elevated testosterone. $^{10}$

A complete blood cell count and comprehensive metabolic panel are also routinely done. Because many people are on restricted diets, a nutrient screen is suggested that includes iron saturation, ferritin, zinc, and vitamin D levels.

\section{DIFFERENTIAL DIAGNOSIS}

Other forms of alopecia that may present similarly to male and female pattern hair loss include telogen effluvium, alopecia areata, traction alopecia, trichotillomania, central centrifugal cicatricial alopecia, lichen planopilaris, and frontal fibrosing alopecia (Figure 3). 15,16

Telogen effluvium, a condition of noninflammatory, diffuse hair loss, is often difficult to distinguish from female pattern hair loss. A thorough history is very important, as there is generally an inciting trigger such as psychological stress, childbirth, weight loss, or medications (eg, interferons, antihyperlipidemic medications, derivatives of retinol, anticoagulants) that precedes telogen effluvium by a few months. Hair loss generally occurs over the entire scalp, occasionally most prominently in the temporal areas. The hair-pull test is positive, with increased shedding of telogen hairs when telogen effluvium is active. Of note, telogen effluvium and female pattern hair loss can coexist in the same patient.

Alopecia areata commonly presents as focal, smooth patches of hair loss, which spontaneously regrow (Figure 3A). Rarely, it can present as diffuse hair loss with widespread decreased hair density (diffuse alopecia areata) or as larger patches of hair loss on the frontal, parietal, and temporal scalp (ophiasis inversus), mimicking female and male pattern hair loss, respectively. Alopecia areata totalis and alopecia areata universalis are characterized by more severe hair loss; alopecia areata totalis represents total loss of scalp hair, whereas alopecia universalis has more extensive hair loss including the face and body in addition to the scalp (Figure 3B).

The onset is usually sudden with prominent shedding, characteristically an anagen effluvium with dystrophic anagen hairs. Telogen hairs are typically lost during chronic shedding. These patients commonly have a positive family history of alopecia areata. ${ }^{17}$ Additionally, nail involvement, such as pitting, longitudinal fissuring, and lunula reddening, occur in $10 \%$ to $20 \%$ of patients with alopecia areata. ${ }^{18}$ The hair-pull test is positive in patients who are actively shedding.

Traction alopecia is a result of chronic (prolonged or repeated) tension on the hair, often from hairstyles. Hair loss along the hairline is common (Figure 3C). A thorough his- 



Figure 3. Differential diagnosis for pattern hair loss. A, patchy alopecia areata; B, alopecia totalis; C, traction alopecia; D, trichotillomania; E, central centrifugal cicatricial alopecia; F, lichen planopilaris; G, frontal fibrosing alopecia.

tory can be helpful with the diagnosis.

Trichotillomania is a psychiatric condition in which patients repeatedly pull at their hair. Hair loss can occur on different portions of the body with hairs of different lengths as a result of episodes of hair pulling or variations in breakage point along the hair shaft within the same episode. Hair loss occurs in bizarre patterns (Figure 3D). The eyebrows, eyelashes, and pubic hair can be involved.

Central centrifugal cicatricial alopecia, most commonly affecting women of African descent, is a form of scarring alopecia that often affects the vertex of the scalp. It is associated with the gene PAD13, which encodes an enzyme, type III peptidyl arginine deiminase, critical for hair shaft formation. ${ }^{19}$

Usually, central centrifugal cicatricial alopecia first presents as a patch of hair thinning that progresses to more severe hair loss expanding from the center of the lesion. It increases in size in a centrifugal fashion, with the center most severely affected with loss of hair follicles
(Figure 3E). Central centrifugal cicatricial alopecia can be differentiated from female pattern hair loss by visible loss of the follicular ostia. Additionally, it can present with other signs and symptoms on the scalp, including pustules, erythema, tenderness, and pruritus.

Lichen planopilaris and frontal fibrosing alopecia are uncommon inflammatory scarring alopecias that have similar histologic findings but dissimilar clinical presentations. Classic lichen planopilaris presents as small to large areas of patchy hair loss, frequently affecting the vertex or parietal scalp (Figure 3F). Hair loss in lichen planopilaris may present similarly to central centrifugal cicatricial alopecia, but there are no vellus hairs in lichen planopilaris. ${ }^{20}$ Nail, cutaneous, and mucosal involvement can occur.

Frontal fibrosing alopecia results in recession of the hairline in a bandlike distribution in women (Figure 3G), with perifollicular erythema, and follicular hyperkeratosis. Unlike female pattern hair loss, frontal fibrosing alo- 


\section{TABLE 1}

\section{Workup of pattern hair loss in men and women}

\section{History}

Age of onset, time course, severity, hair loss distribution, progression (periods of shedding), accompanying symptoms

Medical history

Gynecologic history

Medication and supplement list

Family history of hair loss, androgen excess, and metabolic disease

\section{Physical examination \\ Distribution of hair loss \\ Hair caliber and texture \\ Clinical features of scalp \\ Nail changes}

Hair-pull test

\section{Dermoscopy}

\section{Scalp biopsy}

\section{Laboratory testing}

Complete blood cell count

Comprehensive metabolic panel

Antithyroid antibody testing

Thyroid-stimulating hormone with or without free thyroxine

Iron evaluation (serum ferritin, serum iron, total iron-binding capacity)

Free and/or total testosterone

Dehydroepiandrosterone sulfate

Serum prolactin

Zinc

Hydroxy-25 vitamin D pecia often affects the eyebrows and temporal scalp and can result in complete and permanent hair loss.

\section{LOWER SELF-ESTEEM}

Pattern hair loss can lead to negative feelings in both men and women and can contribute to stress, decrease body image satisfaction, damage self-esteem, and diminish quality of life, especially among women and individuals seeking treatment. ${ }^{21}$ Patient and physician perceptions of disease severity may diverge, underlying the importance of attending to the psychosocial and psychoemotional status of these patients. ${ }^{22}$

\section{LOSS IS PROGRESSIVE}

Pattern hair loss is progressive, leaving patients with diminished hair density. Male pattern hair loss can result in complete loss of hair coverage in particular areas, whereas female pattern hair loss rarely advances to baldness.

Response to pharmacologic treatment varies, but it is important to recognize pattern hair loss and initiate treatment early in the disease process to try to prevent further hair loss and promote some degree of hair regrowth.

\section{TREATMENT}

The goal of treatment of pattern hair loss is to promote regrowth of hair to improve scalp coverage and to prevent or slow further hair thinning and loss. Topical minoxidil and oral finasteride are the first-line treatments for male pattern hair loss, and topical minoxidil is the first-line treatment for female pattern hair loss. However, there are a number of alternatives. Detecting and treating comorbidities such as androgen excess and nutritional deficiencies is helpful in maintaining hair growth.

\section{Minoxidil}

Mechanism of action. Through an unclear mechanism, minoxidil enhances hair growth by enlarging miniaturized hair follicles, extending anagen, and shortening telogen. ${ }^{23}$

US Food and Drug Administration (FDA) approval. Minoxidil is FDA-approved for treating both male and female pattern hair loss.

Administration. Topical minoxidil is available over the counter in $2 \%$ and $5 \%$ solutions and 5\% foam in the United States. Patients should apply $1 \mathrm{~mL}$ of $5 \%$ solution or half a cap of $5 \%$ foam once a day directly to involved areas of the scalp (not the hair) when dry. Treatment should be assessed for efficacy after 1 year of use, but results may be observed sooner.

Efficacy in men. In a 48-week, doubleblind, placebo-controlled, randomized trial, 5\% minoxidil solution was superior to $2 \%$ solution in terms of patient perception of hair growth and treatment benefit, investigator perception of hair growth, and nonvellus hair count. ${ }^{24} \mathrm{Re}$ sponse time was also shorter with the $5 \%$ solution. Both the 2\% and the 5\% topical minoxidil solutions were superior to placebo.

Foam has not been directly compared with liquid solutions. Compared with placebo, 5\% minoxidil foam increased the hair count and improved hair loss as assessed by the patient 
and physician. ${ }^{25}$

Individual response to treatment with minoxidil varies, and hair regrowth can be lost after stopping.

Efficacy in women. A meta-analysis found that topical minoxidil was effective and safe for treating female pattern hair loss, with no significant difference in efficacy and safety between different concentrations. ${ }^{26}$ Topical minoxidil is an effective treatment for women regardless of androgen status and age. ${ }^{10}$

Side effects. Minoxidil stimulates telogen follicles to enter anagen, so transient hair shedding may occur when initiating therapy. Contact dermatitis and hypertrichosis are common side effects. Compared with 2\% topical minoxidil, 5\% preparations are more likely to cause local pruritus and irritation. ${ }^{25}$

\section{Finasteride}

Mechanism of action. Finasteride, a competitive inhibitor of type II 5-alpha reductase, decreases production of dihydroxytestosterone. Oral finasteride reduces scalp dihydroxytestosterone levels by approximately $60 \%$ to $70 \%$, depending on the dosage. ${ }^{26}$

FDA approval. Finasteride is FDA-approved for treating male pattern hair loss, but not female pattern hair loss.

Administration. Men with male pattern hair loss can be treated with finasteride $1 \mathrm{mg}$ daily. Treatment should be assessed for efficacy after 1 year of use, but results may be observed sooner.

Efficacy in men. Finasteride has been shown to increase the hair count, physicianassessed hair coverage, and hair mass compared with placebo..$^{27,28}$

A systematic review of the efficacy of finasteride in men with male pattern hair loss found that 5.6 patients need to be treated short-term, and 3.4 patients need to be treated long-term, for 1 patient to perceive an improvement. ${ }^{27}$ There was a $20 \%$ absolute increase in patient-perceived improvement in the short term and a 30\% absolute increase in the long term. ${ }^{27}$ Longer treatment with finasteride promotes greater therapeutic success.

Similar to minoxidil, response to finasteride varies, and hair regrowth can be lost after the medication is discontinued.

Efficacy in women. A double-blind, placebo-controlled, randomized multicenter trial found finasteride $1 \mathrm{mg}$ to be ineffective in postmenopausal women with female pattern hair loss at 12 months. ${ }^{29}$ However, higher doses of finasteride daily can be effective in cases of female pattern hair loss associated with hyperandrogenemia. ${ }^{30}$

Side effects. In a systematic review, the only adverse effect associated with finasteride treatment was erectile dysfunction, with an absolute increase in risk of approximately $1.5 \% .{ }^{27}$ Approximately 1 in every 80 men treated with finasteride experiences this side effect. Stopping finasteride generally leads to resolution of erectile dysfunction, but sexual dysfunction can persist in some patients.

Other reported side effects of finasteride include decreased libido, gynecomastia, testicular pain, and depression.

Considerations. Finasteride can result in lower prostate-specific antigen levels in men, which should be taken into consideration when interpreting laboratory results. Due to hepatic metabolism of finasteride, precautions should be taken with patients with liver disease. With chronic treatment, finasteride may cause mild to moderate elevations in serum liver enzymes, but this is usually self-limiting and rarely requires dose modification or drug discontinuation. ${ }^{31}$

\section{Dutasteride}

Dutasteride, a potent type I and type II 5-alpha reductase inhibitor, is used to treat benign prostatic hyperplasia, but is also prescribed as an off-label treatment for pattern hair loss.

A randomized control trial ${ }^{32}$ in 416 men with male pattern hair loss demonstrated that dutasteride $2.5 \mathrm{mg}$ was superior to finasteride $5 \mathrm{mg}$ in terms of increasing hair count over 24 weeks. Additionally, a meta-analysis ${ }^{33}$ found that finasteride and dutasteride had similar efficacy in treating pattern hair loss.

\section{Latanoprost, bimatoprost}

Latanoprost is a prostaglandin F2 analogue that extends the anagen phase. ${ }^{34}$ A doubleblind, placebo-controlled, randomized trial in 16 men demonstrated an increase in hair density when latanoprost $0.1 \%$ was applied to a small area of the scalp. ${ }^{35}$

Bimatoprost is also being investigated as a potential treatment for pattern hair loss.

\section{Consider conditions that worsen hair loss: eg, iron deficiency, thyroid dysfunction, and nutritional deficiencies}




\section{Ketoconazole, zinc shampoo}

Ketoconazole, an antifungal medication that has anti-inflammatory and antiandrogenetic properties, can be used to treat seborrheic dermatitis and dandruff. ${ }^{36}$ It was found to reduce inflammation in pattern hair loss and to benefit women with hyperandrogenemia and female pattern hair loss when used as a $2 \%$ shampoo. ${ }^{37}$

Over-the-counter zinc-containing shampoos have a similar mechanism of action and therapeutic effect.

\section{Spironolactone}

Spironolactone competitively inhibits the androgen receptor and also inhibits ovarian production of androgens. Of the antiandrogen drugs used off-label, it is the one most commonly used. It has been used to treat female pattern hair loss for over 2 decades and has a good safety profile. ${ }^{37}$ An open-label trial ${ }^{38}$ showed that spironolactone $200 \mathrm{mg}$ daily improved hair regrowth in $44 \%$ of patients at 12 months, which was comparable to cyproterone acetate.

\section{Cyproterone acetate}

Other forms Cyproterone acetate is a progesterone derivative of alopecia may present similarly to male and female pattern hair loss that prevents dihydroxytestosterone from binding to the androgen receptor and inhibits release of follicle-stimulating hormone and luteinizing hormone, thereby reducing testosterone levels. It is available combined with ethynyl estradiol as an oral contraceptive in many countries, but is not approved in the United States.

\section{Platelet-rich plasma}

Platelet-rich plasma is an autologous preparation of plasma with platelets, growth factors, and cytokines. It was initially used during hair transplantation procedures, with mixed results. Recently its use by itself has been explored to treat pattern hair loss. Preliminary evidence suggests that platelet-rich plasma may be advantageous in hair regrowth. ${ }^{39}$ Side effects include redness and pain at injection site and pinpoint bleeding.

\section{Low-level laser therapy}

Low-level laser therapy is an FDA-approved treatment for pattern hair loss. The mechanism by which it improves hair loss is unclear, but it may stimulate follicular stem cells or keratinocytes, increase blood flow, promote mitosis, increase cell metabolism, and have anti-inflammatory effects. ${ }^{40}$ Two doubleblind, sham device-controlled, randomized trials demonstrated that low-level laser therapy increased hair density over 24 to 26 weeks of treatment, although differences global improvement ratings were not significant in one of the trials. ${ }^{41,42}$

\section{Microneedling}

Rolling fine needles over the skin causes minor physical trauma to the stratum corneum that incites wound healing. A randomized controlled trial demonstrated promising results with microneedling as an adjuvant to drug treatment for pattern hair loss. ${ }^{43}$ Side effects of the procedure include pain and pinpoint bleeding.

\section{Cosmetic aids}

Nonmedical options allow patients to manage the appearance of thinning hair. Scalp colorants, including powders, lotions, and hair sprays, can reduce the color contrast between the skin and hair, camouflaging the scalp. The scalp can also be covered with wigs, hair extensions, or hair pieces.

\section{Hair transplants}

Follicles can be transplanted from an unaffected area of the scalp, commonly the occiput, where there are more than 40 follicular units $/ \mathrm{cm}^{3}$, to affected areas to create a permanent improvement in hair coverage. Young patients or patients with vertex involvement are not ideal candidates for this procedure due to the high likelihood of continued progression. ${ }^{10} \mathrm{~A}$ large number of grafts, 1,000 to 2,000 follicles, can be transplanted in a single session. Additional transplantation sessions are generally scheduled at least 6 months apart, as it takes 5 to 6 months for results to be established. ${ }^{10}$

Pharmacologic treatment, such as oral finasteride, can be used in conjunction with hair transplantation before and after sessions to prevent hair miniaturization and loss of nontransplanted hair follicles. ${ }^{44}$

\section{DISCLOSURES}

The authors report no relevant financial relationships which, in the context of their contributions, could be perceived as a potential conflict of interest. 


\section{REFERENCES}

1. Yip L, Rufaut N, Sinclair R. Role of genetics and sex steroid hormones in male androgenetic alopecia and female pattern hair loss: an update of what we now know. Australas J Dermatol 2011; 52(2):81-88. doi:10.1111/j.1440-0960.2011.00745.x

2. Yip L, Zaloumis S, Irwin D, et al. Gene-wide association study between the aromatase gene (CYP19A1) and female pattern hair loss. Br J Dermatol 2009; 161(2):289-294. doi:10.1111/j.1365-2133.2009.09186.

3. Sawaya ME, Price VH. Different levels of 5alpha-reductase type I and II, aromatase, and androgen receptor in hair follicles of women and men with androgenetic alopecia. J Invest Dermatol 1997; 109(3):296-300. doi:10.1111/1523-1747.ep12335779

4. Vierhapper H, Nowotny P, Maier H, WaldhäusI W. Production rates of dihydrotestosterone in healthy men and women and in men with male pattern baldness: determination by stable isotope/dilution and mass spectrometry. J Clin Endocrinol Metab 2001; 86(12):5762-5764. doi:10.1210/jcem.86.12.8078

5. Imperato-McGinley J, Zhu YS. Androgens and male physiology the syndrome of 5alpha-reductase-2 deficiency. Mol Cell Endocrinol 2002; 198(1-2):51-59. doi:10.1016/s0303-7207(02)00368-4

6. Futterweit W, Dunaif A, Yeh HC, Kingsley P. The prevalence of hyperandrogenism in 109 consecutive female patients with diffuse alopecia. J Am Acad Dermatol 1988; 19(5 pt 1):831-836. doi:10.1016/s0190-9622(88)70241-8

7. Cousen P, Messenger A. Female pattern hair loss in complete androgen insensitivity syndrome. Br J Dermatol 2010; 162(5):1135-1137. doi:10.1111/j.1365-2133.2010.09661.x

8. Norwood OT. Male pattern baldness: classification and incidence. South Med J 1975; 68(11):1359-1365. doi:10.1097/00007611-197511000-00009

9. Olsen EA. The midline part: an important physical clue to the clinical diagnosis of androgenetic alopecia in women. J Am Acad Dermatol 1999; 40(1):106-109. doi:10.1016/s0190-9622(99)70539-6

10. Olsen EA, Messenger AG, Shapiro J, et al. Evaluation and treatment of male and female pattern hair loss. J Am Acad Dermatol 2005; 52(2):301-311. doi:10.1016/j.jaad.2004.04.008

11. Ludwig E. Classification of the types of androgenetic alopecia (common baldness) occurring in the female sex. Br J Dermatol 1977; 97(3):247-254. doi:10.1111/j.1365-2133.1977.tb15179.x

12. Piérard-Franchimont $C$, Piérard GE. Approche physiopathologique de la séborrhée du cuir chevelu. Ann Dermatol Venereol 1988; 115(4):451-453. French. pmid:2970818

13. McDonald KA, Shelley AJ, Colantonio S, Beecker J. Hair pull test: evidence-based update and revision of guidelines. J Am Acad Dermatol 2017; 76(3):472-477. doi:10.1016/j.jaad.2016.10.002

14. Dhurat R, Saraogi P. Hair evaluation methods: merits and demerits. Int J Trichology 2009; 1(2):108-119. doi:10.4103/0974-7753.58553

15. Miteva M, Tosti A. Hair and scalp dermatoscopy. J Am Acad Dermatol 2012; 67(5):1040-1048. doi:10.1016/j.jaad.2012.02.013

16. Chan L, Cook DK. Female pattern hair loss. Aust J Gen Pract 2018; 47(7):459-464. doi:10.31128/AJGP-02-18-4498

17. Shellow WV, Edwards JE, Koo JY. Profile of alopecia areata: a questionnaire analysis of patient and family. Int J Dermatol 1992; 31(3):186-189. doi:10.1111/j.1365-4362.1992.tb03932.x

18. Strazzulla LC, Wang EHC, Avila L, et al. Alopecia areata: disease characteristics, clinical evaluation, and new perspectives on pathogenesis. J Am Acad Dermatol 2018; 78(1):1-12. doi:10.1016/j.jaad.2017.04.1141

19. Malki L, Sarig O, Romano MT, et al. Variant PADI3 in central centrifugal cicatricial alopecia. N Engl J Med 2019; 380(9):833-841. doi:10.1056/NEJMoa1816614

20. Miteva M, Tosti A. Central centrifugal cicatricial alopecia presenting with irregular patchy alopecia on the lateral and posterior scalp. Skin Appendage Disord 2015; 1(1):1-5. doi:10.1159/000370315

21. Cash TF. The psychosocial consequences of androgenetic alopecia: a review of the research literature. Br J Dermatol 1999; 141(3):398 405. doi:10.1046/j.1365-2133.1999.03030.x
22. Reid EE, Haley AC, Borovicka JH, et al. Clinical severity does not reliably predict quality of life in women with alopecia areata, telogen effluvium, or androgenic alopecia. J Am Acad Dermatol 2012; 66(3):e97-e102. doi:10.1016/j.jaad.2010.11.042

23. Messenger AG, Rundegren J. Minoxidil: mechanisms of action on hair growth. Br J Dermatol 2004; 150(2):186-194. doi:10.1111/j.1365-2133.2004.05785.x

24. Olsen EA, Dunlap FE, Funicella T, et al. A randomized clinical trial of $5 \%$ topical minoxidil versus $2 \%$ topical minoxidil and placebo in the treatment of androgenetic alopecia in men. J Am Acad Dermatol 2002; 47(3):377-385. doi:10.1067/mjd.2002.124088

25. Olsen EA, Whiting D, Bergfeld W, et al. A multicenter, randomized, placebo-controlled, double-blind clinical trial of a novel formulation of $5 \%$ minoxidil topical foam versus placebo in the treatment of androgenetic alopecia in men. J Am Acad Dermatol 2007; 57(5):767774. doi:10.1016/j.jaad.2007.04.012

26. van Zuuren EJ, Fedorowicz Z, Schoones J. Interventions for female pattern hair loss. Cochrane Database Syst Rev 2016; 2016(5):CD007628. doi:10.1002/14651858.CD007628.pub4

27. Mella JM, Perret MC, Manzotti M, Catalano HN, Guyatt G. Efficacy and safety of finasteride therapy for androgenetic alopecia: a systematic review. Arch Dermatol 2010; 146(10):1141-1150. doi:10.1001/archdermatol.2010.256

28. Adil A, Godwin M. The effectiveness of treatments for androgenetic alopecia: a systematic review and meta-analysis. J Am Acad Dermatol 2017; 77(1):136-141.e5. doi:10.1016/j.jaad.2017.02.054

29. Price VH, Roberts JL, Hordinsky M, et al. Lack of efficacy of finasteride in postmenopausal women with androgenetic alopecia. J Am Acad Dermatol 2000; 43(5 pt 1):768-776. doi:10.1067/mjd.2000.107953

30. Shum KW, Cullen DR, Messenger AG. Hair loss in women with hyperandrogenism: four cases responding to finasteride. J Am Acad Dermatol 2002; 47(5):733-739. doi:10.1067/mjd.2002.124608

31. Alpha reductase inhibitors. In: LiverTox: Clinical and Research Information on Drug-Induced Liver Injury [Internet]. Bethesda (MD): National Institute of Diabetes and Digestive and Kidney Diseases; 2012. Updated January 9, 2018. pmid: 31644067

32. Olsen EA, Hordinsky M, Whiting $D$, et al. The importance of dual 5alpha-reductase inhibition in the treatment of male pattern hair loss: results of a randomized placebo-controlled study of dutasteride versus finasteride. J Am Acad Dermatol 2006; 55(6):1014-1023. doi:10.1016/j.jaad.2006.05.007

33. Gupta AK, Charrette A. The efficacy and safety of 5a-reductase inhibitors in androgenetic alopecia: a network meta-analysis and benefit-risk assessment of finasteride and dutasteride. J Dermatolog Treat 2014; 25(2):156-161. doi:10.3109/09546634.2013.813011

34. Valente Duarte de Sousa IC, Tosti A. New investigational drugs for androgenetic alopecia. Expert Opin Investig Drugs 2013; 22(5):573589. doi:10.1517/13543784.2013.784743

35. Blume-Peytavi U, Lönnfors S, Hillmann K, Garcia Bartels N. A randomized double-blind placebo-controlled pilot study to assess the efficacy of a 24 -week topical treatment by latanoprost $0.1 \%$ on hair growth and pigmentation in healthy volunteers with androgenetic alopecia. J Am Acad Dermatol 2012; 66(5):794-800. doi:10.1016/j.jaad.2011.05.026

36. Inui S, Itami S. Reversal of androgenetic alopecia by topical ketoconzole: relevance of anti-androgenic activity. J Dermatol Sci 2007; 45(1):66-68. doi:10.1016/j.jdermsci.2006.08.011

37. Kelly Y, Blanco A, Tosti A. Androgenetic alopecia: an update of treatment options. Drugs 2016; 76(14):1349-1364. doi:10.1007/s40265-016-0629-5

38. Sinclair R, Wewerinke M, Jolley $\mathbf{D}$. Treatment of female pattern hair loss with oral antiandrogens. Br J Dermatol 2005; 152(3):466-473. doi:10.1111/j.1365-2133.2005.06218.x

39. Schiavone G, Raskovic D, Greco J, Abeni D. Platelet-rich plasma for androgenetic alopecia: a pilot study. Dermatol Surg 2014; 40(9):1010-1019. doi:10.1097/01.DSS.0000452629.76339.2b

40. Jimenez JJ, Wikramanayake TC, Bergfeld W, et al. Efficacy and safety of a low-level laser device in the treatment of male and fe- 


\section{HAIR LOSS}

male pattern hair loss: a multicenter, randomized, sham device-controlled, double-blind study. Am J Clin Dermatol 2014; 15(2):115-127. doi:10.1007/s40257-013-0060-6

41. Kim H, Choi JW, Kim JY, Shin JW, Lee SJ, Huh CH. Low-level light therapy for androgenetic alopecia: a 24-week, randomized, doubleblind, sham device-controlled multicenter trial. Dermatol Surg 2013; 39(8):1177-1183. doi:10.1111/dsu.12200

42. Leavitt M, Charles G, Heyman E, Michaels D. HairMax LaserComb laser phototherapy device in the treatment of male androgenetic alopecia: a randomized, double-blind, sham device-controlled, multicentre trial. Clin Drug Investig 2009; 29(5):283-292. doi:10.2165/00044011-200929050-00001

43. Dhurat R, Sukesh M, Avhad G, Dandale A, Pal A, Pund P. A randomized evaluator blinded study of effect of microneedling in androgenetic alopecia: a pilot study. Int J Trichology 2013; 5(1):6-11. doi:10.4103/0974-7753.114700

44. Leavitt M, Perez-Meza D, Rao NA, Barusco M, Kaufman KD, Ziering C. Effects of finasteride (1 mg) on hair transplant. Dermatol Surg 2005; 31(10):1268-1276. doi:10.1111/j.1524-4725.2005.31202

Address: Nina L. Tamashunas, BS, Case Western Reserve University School of Medicine, 9501 Euclid Avenue, Cleveland, OH 44106; nlt23@case.edu 